\title{
Recent Innovations in Muon Beam Cooling *
}

\author{
Rolland P. Johnson ${ }^{\epsilon c}$, Mohammad Alsharo'a ${ }^{c}$, Charles Ankenbrandt ${ }^{a}$, \\ Emanuela Barzi ${ }^{a}$, Kevin Beard ${ }^{d}$, S. Alex Bogacz ${ }^{d}$, Yaroslav Derbenev ${ }^{d}$, \\ Licia Del Frate ${ }^{a}$, Ivan Gonin ${ }^{a}$, Pierrick M. Hanlet ${ }^{c}$, Robert Hartline ${ }^{c}$, \\ Daniel M. Kaplan ${ }^{b}$, Moyses Kuchnir ${ }^{c}$, Alfred Moretti ${ }^{a}$, David Neuffer ${ }^{a}$, \\ Kevin Paul ${ }^{c}$, Milorad Popovic ${ }^{a}$, Thomas J. Roberts ${ }^{c}$, Gennady Romanov $^{a}$, \\ Daniele Turrioni ${ }^{a}$, Victor Yarba ${ }^{a}$, Katsuya Yonehara ${ }^{b c}$, \\ ${ }^{a}$ Fermi National Accelerator Laboratory, Batavia, Illinois, U.S.A. \\ ${ }^{b}$ Illinois Institute of Technology, Chicago, Illinois, U.S.A. \\ ${ }^{c}$ Muons, Inc., Batavia, Illinois, U.S.A. \\ ${ }^{d}$ Thomas Jefferson National Accelerator Facility, Newport News, Virginia, U.S.A.
}

\begin{abstract}
Eight new ideas are being developed under SBIR/STTR grants to cool muon beams for colliders, neutrino factories, and muon experiments. Analytical and simulation studies have confirmed that a six-dimensional (6D) cooling channel based on helical magnets surrounding RF cavities filled with dense hydrogen gas can provide effective beam cooling. This helical cooling channel (HCC) has solenoidal, helical dipole, helical quadrupole, and helical sextupole magnetic fields to generate emittance exchange and achieve 6D emittance reduction of over 3 orders of magnitude in a $100 \mathrm{~m}$ segment. Four such sequential HCC segments, where the RF frequencies are increased and transverse physical dimensions reduced as the beams become cooler, implies a $6 \mathrm{D}$ emittance reduction of almost five orders of magnitude. Two new cooling ideas, Parametricresonance Ionization Cooling and Reverse Emittance Exchange, then can be employed to reduce transverse emittances to a few mm-mr, which allows high luminosity with fewer muons than previously imagined. We describe these new ideas as well as a new precooling idea based on a HCC with $z$ dependent fields that can be used as MANX, an exceptional 6D cooling demonstration experiment.
\end{abstract}

Keywords: Muon Beam Cooling, Helical Magnet, Muon Collider

PACS: 29.27.-a, 29.20.-c, 14.60.Ef, 41.85.Lc

\section{INTRODUCTION}

The eight ideas discussed below are to improve the cooling of intense muon beams, which could reduce costs of neutrino factories and facilitate designs of highluminosity muon colliders. These ideas have initiated Small Business Innovation Research (SBIR) and Small business Technology Transfer (STTR) projects that represent a coherent program to develop high intensity, low emittance muon beams.

\section{HIGH-PRESSURE RF CAVITIES}

A gaseous energy absorber enables an entirely new technology to generate high accelerating gradients for muons by using the high-pressure region of the Paschen 
curve [1]. This idea of filling RF cavities with gas is new for particle accelerators and is only possible for muons because they do not scatter as do strongly interacting protons or shower as do less-massive electrons. Measurements by Muons, Inc. and IIT at Fermilab have demonstrated that hydrogen gas suppresses RF breakdown very well, about a factor six better than helium at the same temperature and pressure. Consequently, much more gradient is possible in a hydrogen-filled RF cavity than is needed to overcome the ionization energy loss, provided one can supply the required RF power. Hydrogen is also twice as good as helium in ionization cooling effectiveness, viscosity, and heat capacity. Future research efforts will include tests of pressurized RF Cavities in magnetic fields and high radiation environments and the use of new cavity construction materials [2], including beryllium RF windows for improved cavity performance [3].

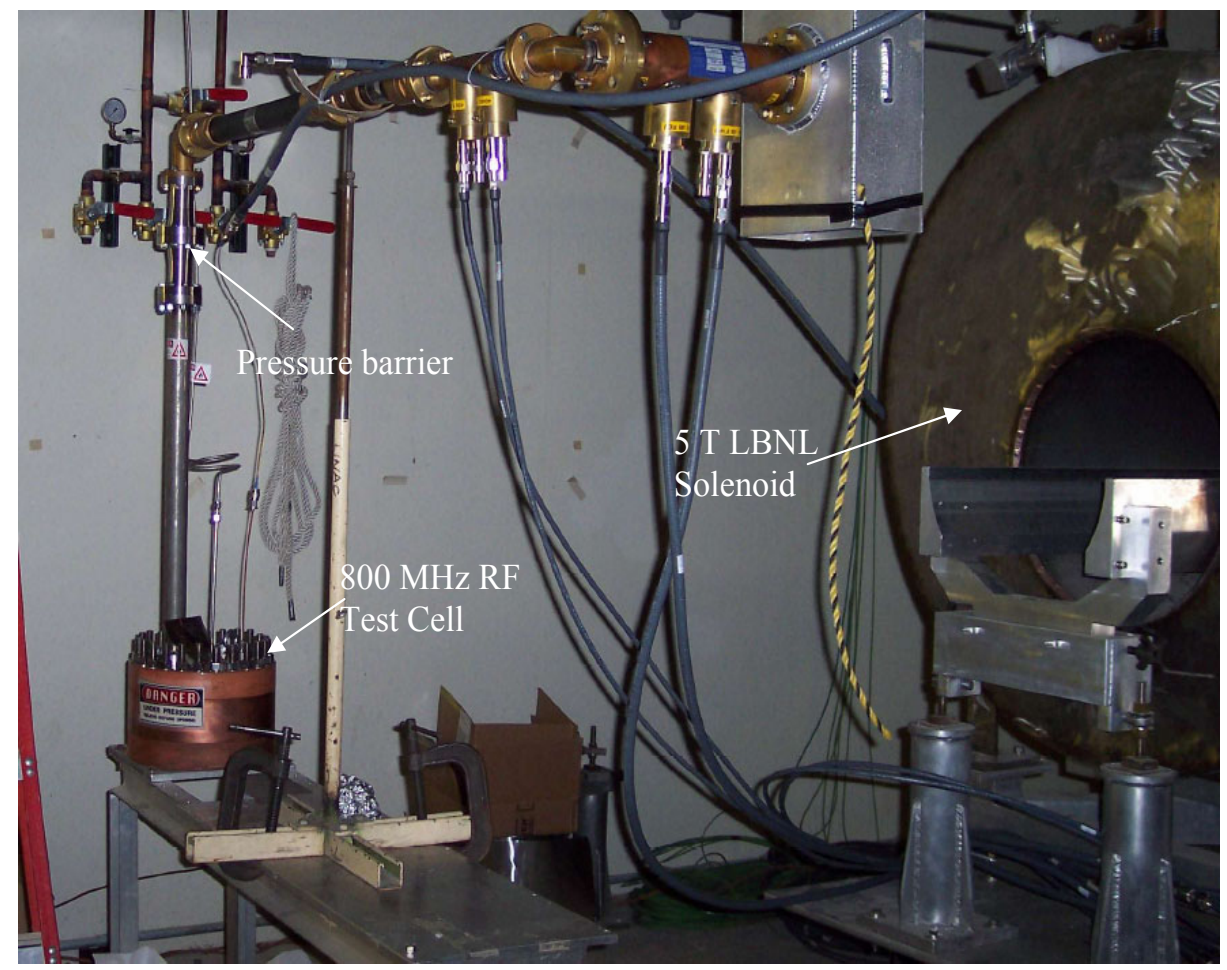

FIGURE 1. MuCool Test Area view of $800 \mathrm{MHz}$ hydrogen-filled high-pressure test cell. The cell is capable of testing RF breakdown of gases up to 2000 PSI and down to $77 \mathrm{~K}$. Future tests include operation of the test cell in the $5 \mathrm{~T}$ solenoidal magnet shown at the right of the picture and in the extracted $400 \mathrm{MeV}$ H- Linac beam, which should be available in 2006.

\section{COMBINED CAPTURE, PHASE ROTATION, AND COOLING}

High-pressure RF cavities near the pion production target can be used to simultaneously capture, bunch rotate, and cool the muon beam as it emerges from the decaying pions [4]. We have started an R\&D effort to develop RF cavities that will 
operate in the extreme conditions near a production target and an effort to simulate the simultaneous capture, phase rotation, and cooling of muons as they are created from pion decay.

\section{EMITTANCE EXCHANGE IN A CONTINUOUS ABSORBER}
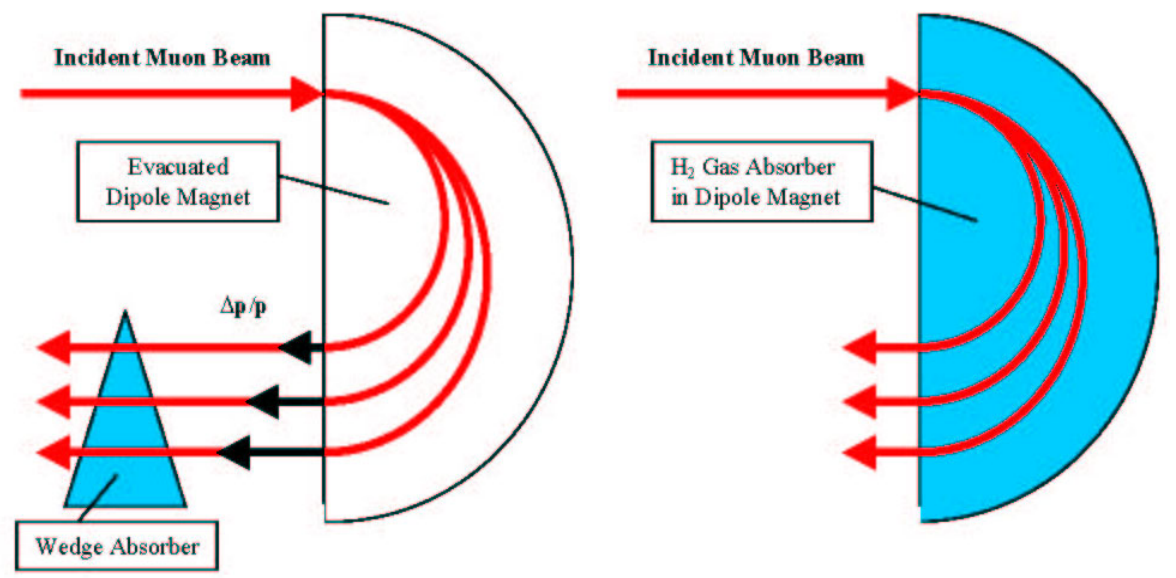

FIGURES 2 (LEFT) AND 3 (RIGHT). Comparison of the usual method of emittance exchange based on a wedge absorber (Left) with the method advocated in this paper using a continuous energy absorber as is possible with pressurized RF cavities (Right).

Ionization Cooling is only transverse. To get $6 \mathrm{D}$ cooling, emittance exchange between transverse and longitudinal coordinates is needed. Figure 2 shows the usual mechanism based on a wedge absorber to achieve reduction in momentum spread at the expense of increasing transverse emittance. In figure 3, positive dispersion gives higher energy muons larger energy loss due to their longer path length in a low- $Z$ absorber.

\section{HELICAL COOLING CHANNELS}

The idea of pressurized RF cavities led to the concept of a cooling channel filled with a continuous homogeneous absorber. Such a cooling channel provides longitudinal ionization cooling by exploiting the path length (and therefore energy loss) correlation with momentum in a magnetic channel with positive dispersion. Using this approach in a helical cooling channel creates emittance exchange and excellent 6D muon beam cooling.

The mathematical treatment of the original helical cooling channel exploits the fact that the magnitudes of the fields are constant and only the directions of the helical fields change with a constant frequency. By transforming to the rotating frame, a $z$ or time independent Hamiltonian can be formed, which leads to an elegant treatment of the properties of the HCC [5]. The two radial forces from the cross products of the 
muon longitudinal and transverse momenta with the helical dipole and solenoidal fields, respectively, oppose each other in the stable solution. The solution has unusually large acceptance and exactly the required dispersion characteristics. Figure 4 shows the simulation of a series of four such helical channels with a factor of $50,0006 \mathrm{D}$ emittance reduction to demonstrate the idea that the dimensions of the channel and the RF frequency can be reduced as the beam is cooled to improve the cooling efficiency. A program to develop HCC magnets has started at Fermilab [6].

\begin{tabular}{|l|l|l|l|}
\hline$\lambda=1.0 \mathrm{~m}$ & $\lambda=0.8 \mathrm{~m}$ & $\lambda=0.6 \mathrm{~m} \quad \lambda=0.4 \mathrm{~m}$ \\
\hline
\end{tabular}

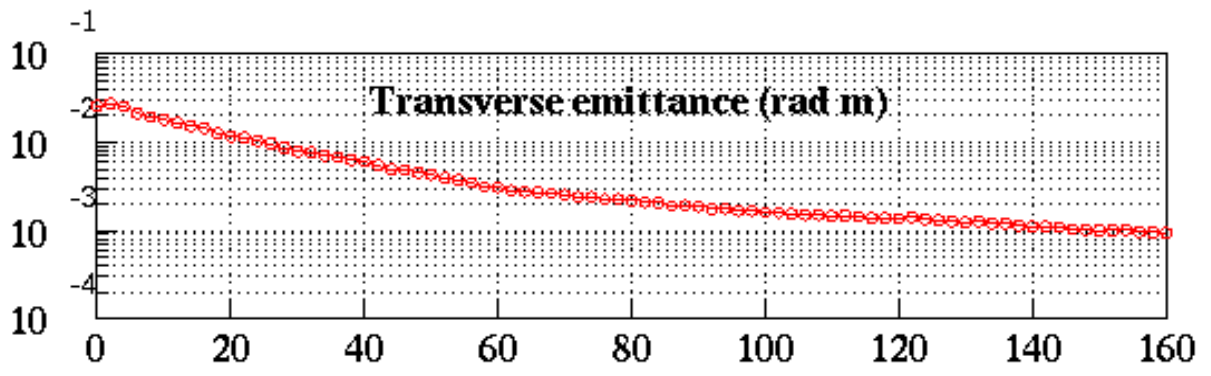
$-1$
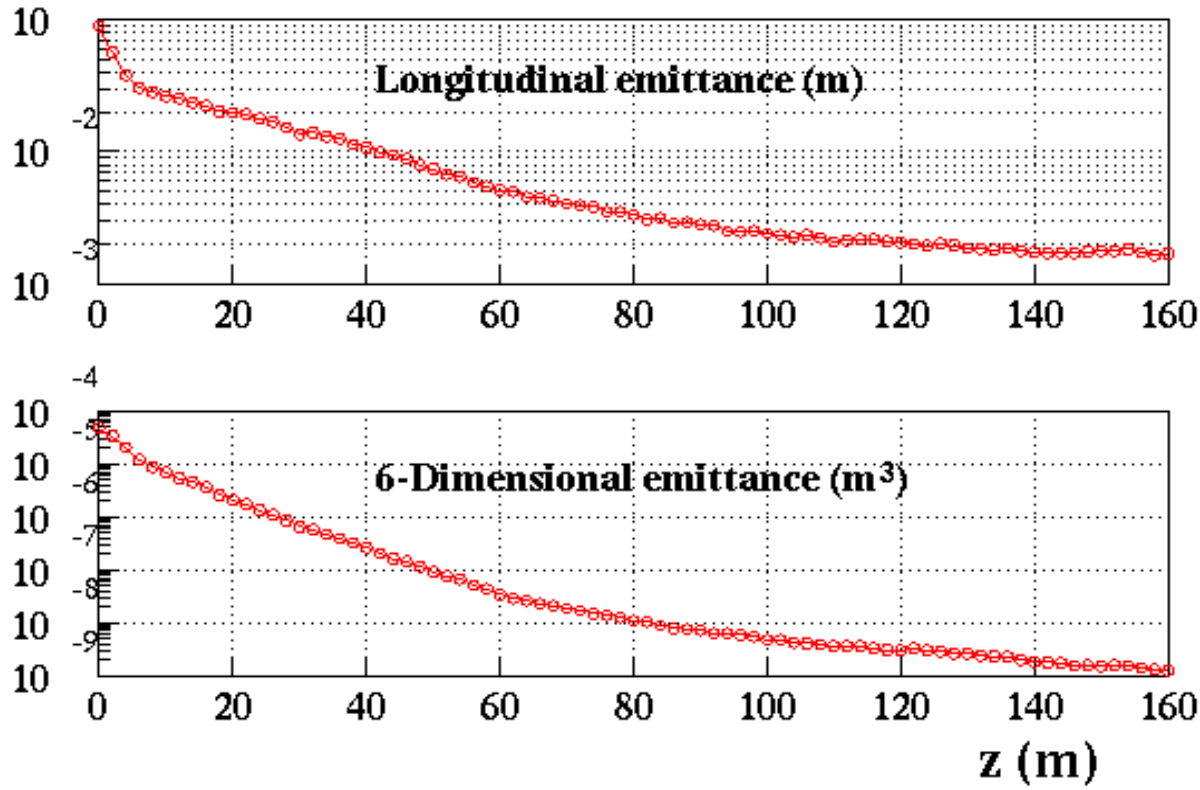

FIGURE 4. Emittance evolution for a series of four helical cooling channels with shorter and shorter helix periods and higher magnetic fields as calculated by the G4Beamline simulation program. Hydrogen-filled $200 \mathrm{MHz}$ RF cavities embedded in solenoidal and helical dipole, quadrupole, and sextupole fields provide continuous 6-dimensional cooling. The simulation uses Maxwellian fields for the magnets. The practical design of devices that can provide these fields is under study. 


\section{MOMENTUM DEPENDENT HCC USES: PRECOOLER}

The solution for a particle in a HCC with period $\lambda=2 \pi / k$ on an equilibrium orbit with radius $a$ and momentum $p$ can be written:

$$
p(a)=\frac{\sqrt{1+\kappa^{2}}}{k}\left[B-\frac{1+\kappa^{2}}{\kappa} b(\kappa)\right],
$$

where $\kappa=k a=p_{\perp} / p_{z}$ is the arctangent of the helix pitch angle at the periodic orbit, $B$ is the magnitude of the solenoidal field and $b$ is the magnitude of the helical dipole field.

The new idea is that this relationship can be exploited in cases where the beam momentum is not constant to provide the required dispersion and orbit for effective cooling. We can use equation (1) to change fields and helix parameters to maintain the orbit and dispersion properties. The HCC concept can thus be extended to have momentum dependent magnetic field strengths for several new applications.

This conceptual change that allowed it to become a $z$ or momentum dependent device has made the HCC a potential work-horse for different components of the muon beam cooling channel. For example, by filling the HCC with hydrogen or helium, the beam can be decelerated and cooled by ionization energy loss over more than $100 \mathrm{MeV} / \mathrm{c}$, then reaccelerated by a series of $\mathrm{RF}$ cavities (pressurized or conventional). The HCC magnet parameters must be varied to match the momentum of the beam as it slows down, according to equation (1). Filled with gas, the HCC with $z$ dependent magnetic field parameters can be used as a transition between HCC sections with different RF frequencies and fields.

Filled with liquid, the HCC with momentum dependent field parameters followed by $\mathrm{RF}$ cavities can be a $6 \mathrm{D}$ precooler, a $6 \mathrm{D}$ demonstration experiment, or an alternative to the original gas-filled HCC (where the momentum is kept almost constant). Two examples discussed below are a 6D precooler and a 6D $\underline{\text { Muon Collider }}$ And Neutrino Factory muon beam cooling demonstration eXpperiment, MANX [7], which is being designed to follow MICE $[8,9]$.

Figure 5 shows a G4BL simulation of a $5 \mathrm{~m}$ HCC precooler that follows a $40 \mathrm{~m}$ $\mathrm{HCC}$ decay channel. Figure 6 shows the normalized transverse (the average radial and azimuthal), longitudinal, and 6D emittances plotted as a function of the distance down the channel. The settings of the helical dipole, helical quadrupole and the solenoidal magnets are chosen to give equal cooling decrements in all three planes. The combined $6 \mathrm{D}$ cooling factor is about 5.4 , corresponding to 1.7 coming from each of the three planes. The improved performance of this precooler simulation relative to previous non-HCC cooling channel designs comes from the effectiveness of HCC, from the greater path length in the hydrogen absorber $\left(5 \mathrm{~m} / \cos \left(45^{\circ}\right)=7.07 \mathrm{~m}\right)$, and from less heating by high- $Z$ windows. The previous designs have aluminum windows on each side of many short liquid hydrogen containment vessels for separation from evacuated RF cavities, while the two thin windows needed for the precooler have not yet been included in the simulation. 

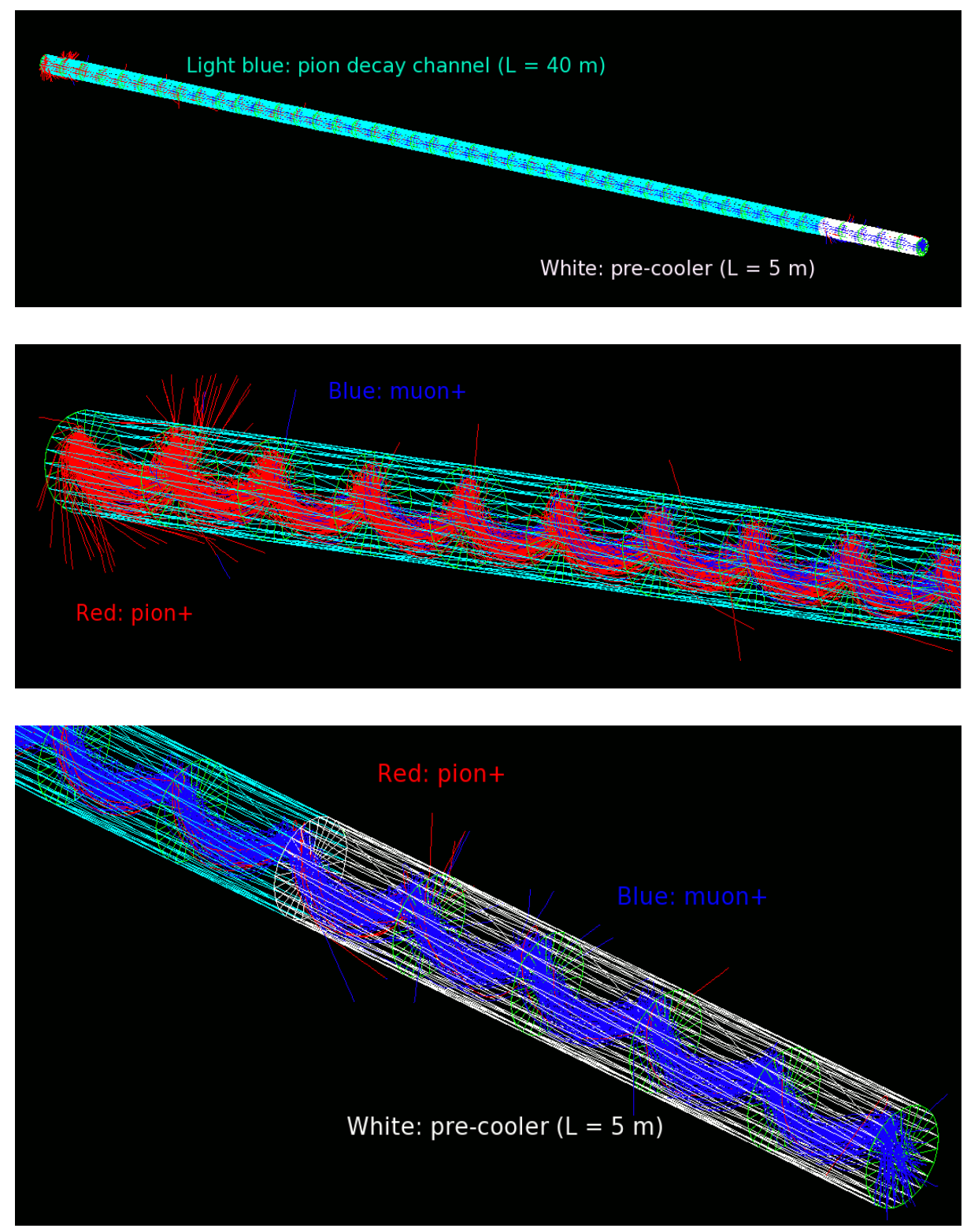

FIGURE 5. Examples of a momentum dependent HCC used as a decay region and as a precooler. The top view shows an overview of a $40 \mathrm{~m}$ HCC used as a pion decay channel followed by a $5 \mathrm{~m}$ liquid hydrogen filled HCC used to precool the muon beam. Pions are shown in red and muons in blue. The center view shows the pion production region. The bottom view shows the liquid hydrogen precooler region of the channel. The emittance evolution of the beam in the precooler is shown in the next figure. 

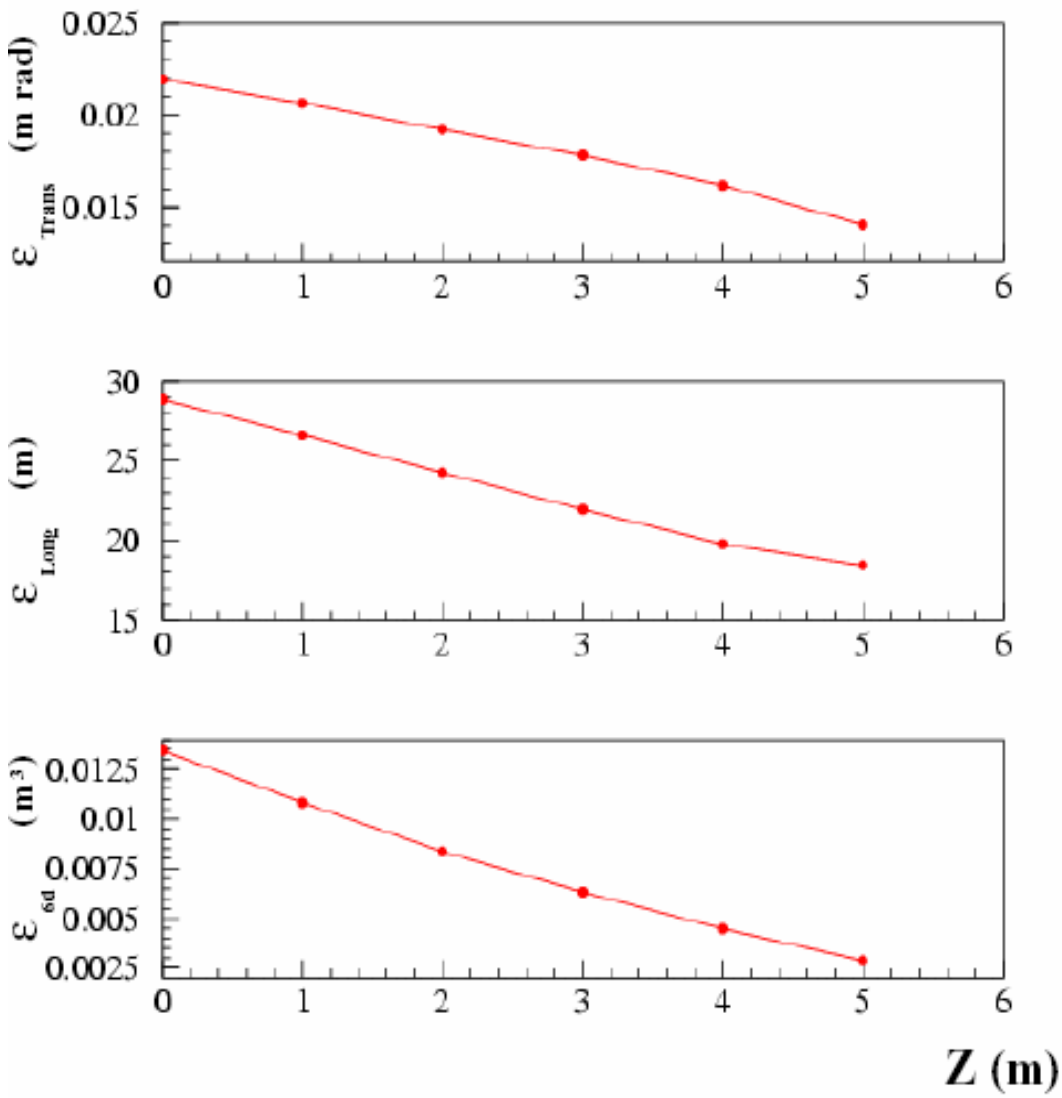

FIGURE 6. The transverse, longitudinal, and 6D emittance evolution of the beam in the precooler.

\section{MANX 6-DIMENSIONAL COOLING DEMONSTRATION}

The excellent cooling of the previous precooler design has inspired a new idea for a MANX 6D demonstration experiment. Namely, we can use a liquid-filled HCC without RF to demonstrate emittance exchange in a prototype precooler. Recent work reported at this workshop [10] has been to optimize the momentum dependent HCC design to work with liquid helium $(\mathrm{LHe})$ instead of liquid hydrogen $\left(\mathrm{LH}_{2}\right)$ for safety reasons and to relax the beam momentum and HCC magnetic field parameters to allow easier construction using available technology. Figure 7 shows the emittance evolution in the new design based on LHe which has a maximum field of $5.5 \mathrm{~T}, 4.5 \mathrm{~m}$ length, and inner diameter of $70 \mathrm{~cm}$.

The factor of 4.2 in $6 \mathrm{D}$ emittance reduction shown in figure 7 is only slightly worse than the factor of 5.5 in the $\mathrm{LH}_{2}$ case. Although each transverse equilibrium emittance should be twice as large for LHe compared to $\mathrm{LH}_{2}$ based on their energy loss and radiation lengths, the cooling factors are very similar when far from equilibrium. 

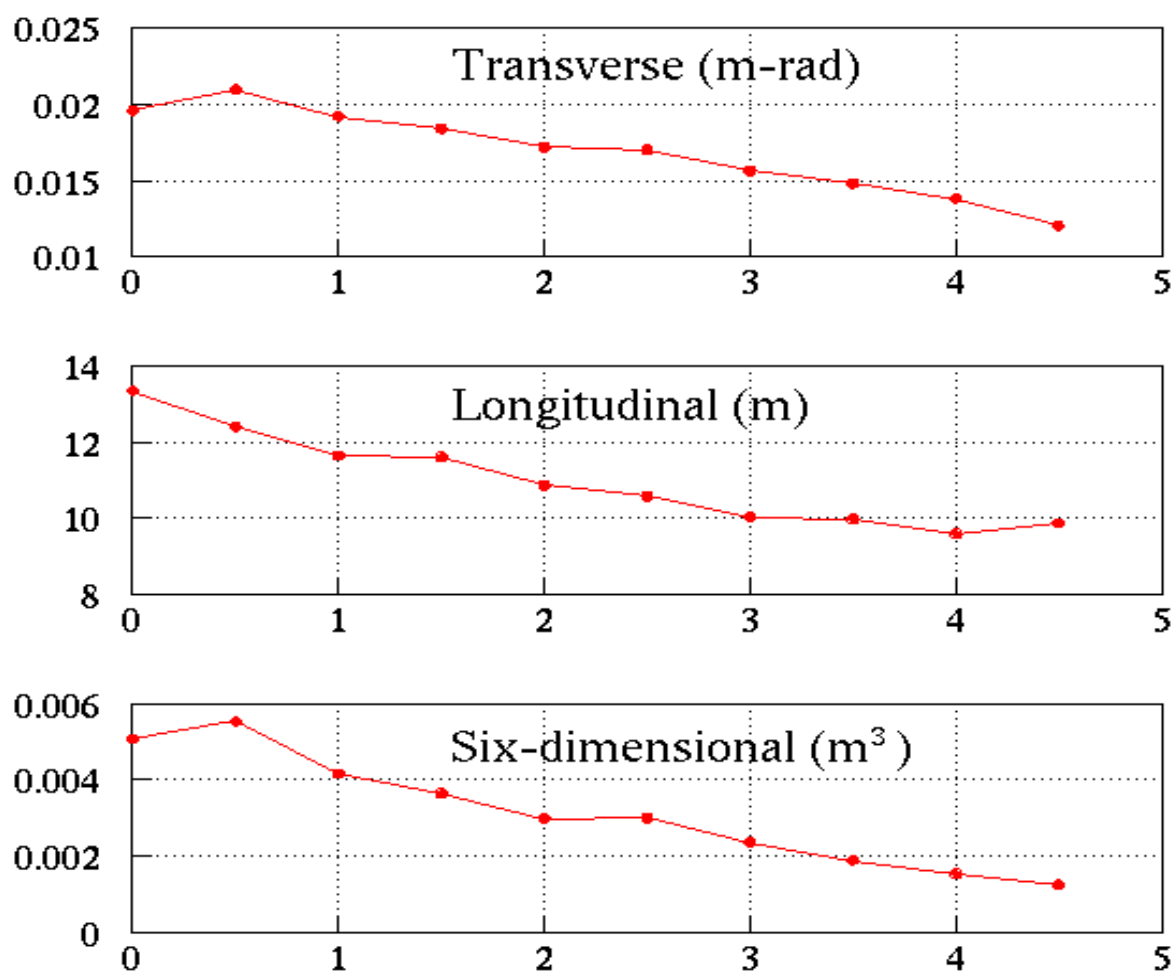

FIGURE 7. The emittance evolution of the beam in a liquid helium HCC designed to have reduced field requirements by using lower initial momentum, longer period, and smaller pitch angle.

\section{PARAMETRIC-RESONANCE IONIZATION COOLING}

To reduce the number of muons required for a high-luminosity muon collider, additional cooling is required beyond what can be accomplished with a HCC even using $20 \mathrm{~T}$ magnets. The large number of muons that have been assumed in previous muon collider plans have been an impediment to acceptance for several reasons: the proton driver and required targetry to produce the required number of muons is difficult, the decay of muons in the collider produces a large electron background in the detectors, and the neutrinos from the same decays can be a site-boundary radiation problem. Additionally, smaller muon beam emittances can mean cost savings in muon acceleration and can allow smaller $\beta^{*}$ at the interaction regions.

Accelerator physicists are used to $1 / 2$-integer parametric resonances as a way to extract beam from a synchrotron. The normal elliptical motion of particles in a beam as shown on the left of figure 8 is transformed into hyperbolic motion by operating on the $1 / 2$-integer resonance as shown on the right of the figure. Thus particles move to larger and larger $x$ as they circulate in a synchrotron until they pass an electrostatic septum and are extracted from the beam. In the scheme that is being developed now for muon cooling beyond the HCC, the perturbations that drive the $1 / 2$-integer 
resonance have a phase that causes the particles to stream the other way such that they go to smaller and smaller $x$ and larger and larger $x^{\prime}$. Ionization cooling is then employed to constrain or shrink the $x^{\prime}$ dimension. Thus the beam size is reduced by the action of the resonance and the angular divergence is reduced by ionization cooling. An essential aspect of this method of cooling, which we anticipate can reduce each transverse dimension another factor of 10, is the control of the aberrations that cause detuning and loss of the resonance condition. Some clever techniques to control the chromatic and spherical aberrations have been developed analytically and are now being simulated.

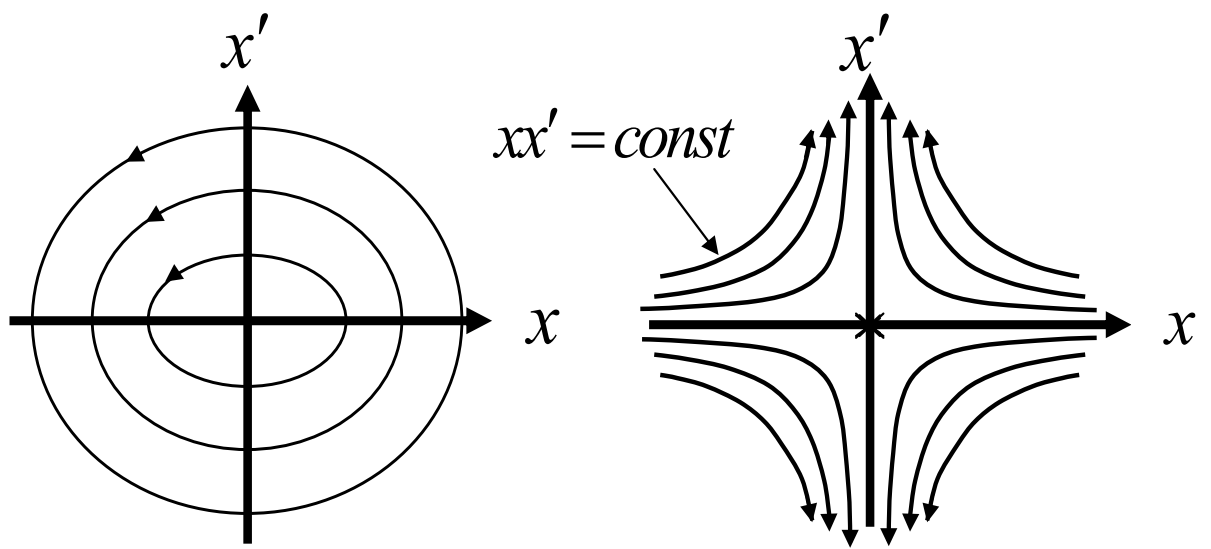

FIGURE 8. Comparison of particle motion at periodic locations along the beam trajectory in transverse phase space for: LEFT ordinary oscillations and RIGHT hyperbolic motion induced by perturbations at a harmonic of the betatron frequency.

\section{REVERSE EMITTANCE EXCHANGE}

Reverse emittance exchange is another technique to reduce the transverse emittances of a muon beam beyond the limits of a HCC in order to increase muon collider luminosity. For example, in the case of a collider of $2.5 \mathrm{TeV} / \mathrm{c}$ on $2.5 \mathrm{TeV} / \mathrm{c}$, the momentum spread $\Delta p / p$ will decrease by a factor of over 10,000 from when it was cooled so that the bunch length will be much shorter than the $\beta^{*}$ of the interaction region.

While longitudinal cooling is essential to reduce the physical dimensions and increase the RF frequency of the cooling and low energy accelerating systems, it will be more than is required for the collider. The new idea is to use wedge absorbers to exchange this unusable longitudinal emittance with the transverse emittance as shown in figure 9. The first analytical treatments of reverse emittance exchange and parametric-resonance ionization cooling have been presented at this conference [11] and have shown that it is possible and perhaps necessary that that these two techniques will be done in the same cooling channel segment. 


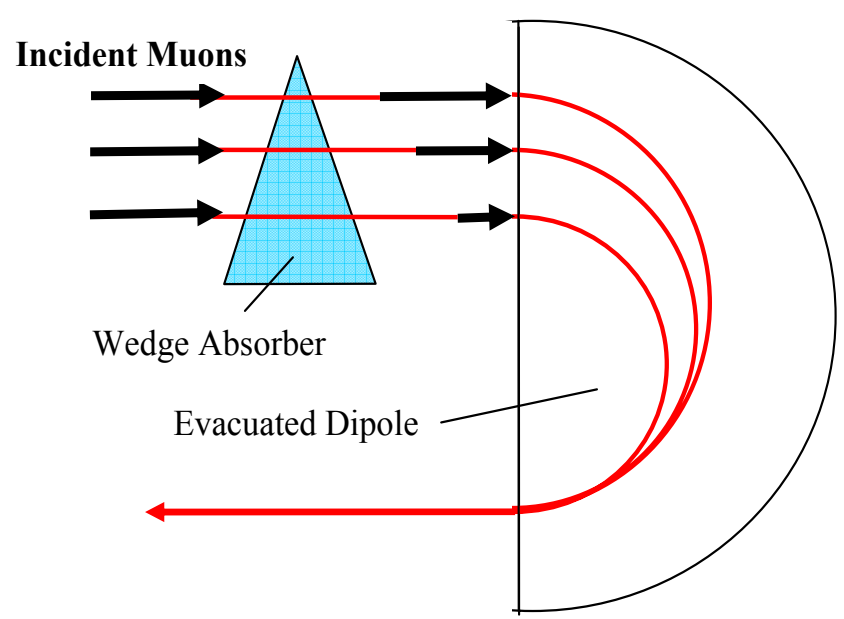

FIGURE 9. Conceptual picture of reverse emittance exchange. A wide beam with narrow momentum spread is transformed using a wedge absorber into a narrow beam with wider momentum spread. The corresponding decrease in transverse emittance leads to higher luminosity while the corresponding increase in longitudinal emittance has little effect on the luminosity as long as the bunch length remains smaller than the focal length of the interaction region.

\section{CONCLUSIONS}

The most exciting development in the last few years has been the realization that there are many new ideas for muon beam cooling. The eight that we have described above may be only the first of many to come that will make muon accelerators and storage rings realistic options for physics research in the near future.

\section{REFERENCES}

\footnotetext{
* Supported by DOE SBIR/STTR grants DE-FG02-02ER86145, 03ER83722, 04ER84015, 04ER86191, and 04ER84016

${ }^{€}$ Corresponding author, rol@muonsinc.com

[1] R. P. Johnson et al. LINAC2004, http://www.muonsinc.com/TU203.pdf

[2] P. M. Hanlet et al., PAC05, http://snsapp1.sns.ornl.gov/pac05/TPPP054/TPPP054.PDF

[3] M. Alsharo'a et al., PAC05, http://snsapp1.sns.ornl.gov/pac05/TPPP053/TPPP053.PDF

[4] K. Paul et al., PAC05, http://snsapp1.sns.ornl.gov/pac05/TPPP055/TPPP055.PDF

[5] Y. Derbenev and R. P. Johnson, Phys.Rev.STAB 8, 041002, (2005)

[6] L. Del Frate et al. PAC05, http://snsapp1.sns.ornl.gov/pac05/TPPP050/TPPP050.PDF

[7] T. J. Roberts et al., PAC05, http://snsapp1.sns.ornl.gov/pac05/TPPP056/TPPP056.PDF

[8] D. M. Kaplan, this workshop

[9] M. A. Cummings et al., this workshop

[10] K. Yonehara, et al., this workshop

[11] Y. Derbenev, this workshop
} 